\title{
He I $\lambda 10830$ AS A PROBE OF WINDS IN ACCRETING YOUNG STARS
}

\author{
Suzan Edwards, ${ }^{1,2,3}$ William Fischer, ${ }^{4}$ John Kwan, ${ }^{4}$ Lynne Hillenbrand, ${ }^{2,5}$ and A. K. Dupree \\ Received 2003 July 21; accepted 2003 October 30; published 2003 November 14
}

\begin{abstract}
He I $\lambda 10830$ profiles acquired with Keck's NIRSPEC for six young low-mass stars with high disk accretion rates (AS 353A, DG Tau, DL Tau, DR Tau, HL Tau, and SVS 13) provide new insight into accretion-driven winds. In four of the stars, the profiles have the signature of resonance scattering, and they possess a deep and broad blueshifted absorption that penetrates more than $50 \%$ into the $1 \mu \mathrm{m}$ continuum over a continuous range of velocities from near the stellar rest velocity to the terminal velocity of the wind, unlike inner wind signatures seen in other spectral features. This deep and broad absorption provides the first observational tracer of the acceleration region of the inner wind and suggests that this acceleration region is situated such that it occults a significant portion of the stellar disk. The remaining two stars also have blue absorption extending below the continuum, although here the profiles are dominated by emission, requiring an additional source of helium excitation beyond resonant scattering. This is likely the same process that produces the emission profiles seen at He I $5876 \AA$.
\end{abstract}

Subject headings: planetary systems: protoplanetary disks — stars: formation — stars: pre-main-sequence — stars: winds, outflows

\section{INTRODUCTION}

Outflows from young stars undergoing disk accretion likely play a vital role in the process of star formation and in the evolution of protoplanetary disks. Although their ubiquity among accreting young stellar objects (YSOs) is well documented, and there is some evidence for a rough proportion of $\dot{M}_{\text {wind }} / \dot{M}_{\text {acc }} \sim 0.1$ (Königl \& Pudritz 2000), the origin of the outflows remains a mystery. Most outflow models rely on the magnetocentrifugal ejection as the heart of the launching mechanism, where rotating fields in the disk fling material along inclined field lines. A distinguishing characteristic among disk wind models is the location in the system where mass loading onto field lines occurs-either from the inner disk over a range of radii (Königl \& Pudritz 2000) or from the radius at which the stellar magnetosphere truncates the disk, lifting most of the accreting material toward the star into magnetic funnel flows and ejecting the rest along opened stellar field lines emerging from this point (Shu et al. 1994). Others have explored the possibility that accretion-driven winds emerge via magnetohydrodynamic acceleration from the star rather than, or in addition to, the disk (Hirose et al. 1997; Kwan \& Tademaru 1988). Each approach has different implications for angular momentum evolution in accretion disk systems, both for the disk, where the dominant mode of angular momentum transport remains unknown, and for the accreting star, where the means of stellar spin-down in the face of accumulation of high angular momentum material is uncertain.

Observational probes of winds in accreting systems have not yet provided decisive evidence of where the wind originates. Either outflowing gas is observed far from the launch site, i.e.,

\footnotetext{
${ }^{1}$ Guest Observer, Gemini/Keck-NIRSPEC program.

${ }^{2}$ Visiting Astronomer, Keck Observatory.

${ }^{3}$ Five College Astronomy, Smith College, McConnell Hall, Northampton, MA 10163; sedwards@smith.edu.

${ }^{4}$ Five College Astronomy Department, University of Massachusetts at Amherst, LGRT-B 622, Amherst, MA, 01003; wfischer@nova.astro.umass.edu, kwan@nova.astro.umass.edu.

${ }^{5}$ Department of Astronomy, California Institute of Technology, Pasadena, CA 91125; lah@astro.caltech.edu.

${ }^{6}$ Harvard-Smithsonian Center for Astrophysics, 60 Garden Street, Cambridge, MA 01238; dupree@cfa.harvard.edu.
}

in collimated jets and molecular outflows, or else inner winds close to the star produce kinematic signatures that are entangled with emission derived from other phenomena such as funnel flows and accretion shocks associated with magnetospheric accretion. Among the classical T Tauri stars (CTTSs), the traditional indicator of an inner wind is blueshifted absorption superposed on strong and broad emission lines, as seen in $\mathrm{H} \alpha$, $\mathrm{Na} \mathrm{D}, \mathrm{Ca}$ II $\mathrm{H}$ and $\mathrm{K}$, and $\mathrm{Mg}$ II $h$ and $k$ (Najita et al. 2000). The line emission is attributed primarily to infall in magnetic funnel flows (Hartmann, Hewett, \& Calvet 1994; Muzerolle, Calvet, \& Hartmann 2001), while the accompanying blueshifted absorption signifies the presence of a high-velocity wind close to the star (Calvet 1997; Ardila et al. 2002). However, little progress has been made in understanding the properties of this inner wind or in establishing whether it originates from a range of radii in the inner disk, the disk truncation radius, or the star.

A recent analysis of profiles of He I $\lambda 5876$ and $\lambda 6678$ in a sample of 31 accreting CTTSs revealed a new means of diagnosing the inner wind (Beristain, Edwards, \& Kwan 2001, hereafter BEK). The helium lines were selected for study because of their high-excitation potential, restricting line formation to a region of either high temperature or close proximity to a source of ionizing radiation. In spite of these restrictions, the helium emission lines were found to have a composite origin, including contributions from a wind, from the funnel flow, and from an accretion shock. The wind component, characterized by broad blueshifted emission (with centroid velocities exceeding $-30 \mathrm{~km} \mathrm{~s}^{-1}$ ) and extended blue wings (with maximum velocities exceeding $-200 \mathrm{~km} \mathrm{~s}^{-1}$ ), was most prominent among stars with the highest disk accretion rates. Curiously, the high accretion rate stars with a strong hot helium wind component were also found to have anomalously weak or absent signatures of the narrow emission component formed in the accretion shock where the magnetosphere directs accreting material from the disk. We interpreted these findings as indicating that a hot accretion-driven wind originates in the vicinity of the stellar corona and that when the hot wind is present, the conditions of magnetospheric accretion and its accompanying accretion shock may be modified.

This intriguing result led us to pursue an investigation of an additional line in the $\mathrm{He}$ I triplet series, $\lambda 10830$, immediately 
TABLE 1

Classical T Tauri Stars

\begin{tabular}{|c|c|c|c|c|c|c|c|c|}
\hline Star & $\begin{array}{c}V_{\star}^{\mathrm{a}} \\
\left(\mathrm{km} \mathrm{s}^{-1}\right)\end{array}$ & $\begin{array}{c}-W_{\lambda}^{\mathrm{b}} \\
(\AA)\end{array}$ & $\begin{array}{c}+W_{\lambda}{ }^{\mathrm{c}} \\
(\AA)\end{array}$ & $\begin{array}{c}\operatorname{Net} W_{\lambda}{ }^{\mathrm{d}} \\
(\AA)\end{array}$ & $\begin{array}{c}V_{\max }^{\mathrm{e}} \\
\left(\mathrm{km} \mathrm{s}^{-1}\right)\end{array}$ & $\begin{array}{c}V_{\text {jet }}^{\mathrm{f}} \\
\left(\mathrm{km} \mathrm{s}^{-1}\right)\end{array}$ & $r_{Y}^{\mathrm{g}}$ & $\begin{array}{c}\log \dot{M}_{\mathrm{acc}}{ }^{\mathrm{h}} \\
\left(M_{\odot} \mathrm{yr}^{-1}\right)\end{array}$ \\
\hline AS 353A & -10 & -3.1 & 4.0 & 0.9 & 300 & 300 & 2.2 & -5.4 \\
\hline DG Tau . & +16 & -1.0 & 8.9 & 7.9 & 450 & 250 & 1.8 & -5.7 \\
\hline DL Tau...$\ldots \ldots$ & +16 & -2.5 & 19.0 & 16.5 & 200 & 200 & 1.0 & -6.7 \\
\hline DR Tau $\ldots \ldots \ldots$ & +22 & -11.3 & 1.1 & -10.2 & 400 & 200 & 2.6 & -5.1 \\
\hline HL Tau ......... & +18 & -4.0 & 2.5 & -1.5 & 200 & 180 & 1.0 & -5.4 \\
\hline SVS $13 \ldots \ldots \ldots$ & +13 & -4.0 & 0 & -4.0 & 250 & 150 & $\ldots$ & -6.2 : \\
\hline
\end{tabular}

${ }^{\mathrm{a}}$ Heliocentric velocity of ambient molecular gas used for stellar rest velocity (from Edwards \& Snell 1982 and Snell \& Edwards 1981).

${ }^{\mathrm{b}}$ Equivalent width below the continuum.

${ }^{\mathrm{c}}$ Equivalent width above the continuum.

${ }^{\mathrm{d}}$ Net equivalent width.

${ }^{\mathrm{e}}$ Maximum velocity of He I 10830 blueshifted absorption.

${ }^{\mathrm{f}}$ Jet radial velocities (from HEG, Mundt et al. 1990, and Davis et al. 2003).

${ }^{\mathrm{g}}$ Veiling in the $Y$ band at $1 \mu \mathrm{m}$.

${ }^{\mathrm{h}}$ Mass accretion rates from HEG and Calvet et al. 1994 and estimated for SVS 13 by assuming the accretion rate is 10 times the published mass-loss rate from Davis et al. 2001.

following $\lambda 5876$ in a recombination-cascade sequence. The lower $2 s^{3} S^{o}$ level of He I $\lambda 10830$ is metastable, resulting in conditions ripe for resonance scattering, which provides an opportunity to search for outflowing gas in absorption. The sensitivity of $\lambda 10830$ absorption to the dynamics of winds in cool stars is illustrated in Dupree, Sasselov, \& Lester (1992). This feature is in a spectral region that, until recently, was not readily accessible to either CCD or infrared imaging detectors. An early study by Ulrich \& Wood (1981), using pre-CCD technology, detected this feature in a few T Tauri stars, but the data were not of sufficient quality to interpret the line profile. The next published work featuring $\lambda 10830$ in a T Tauri star is for DG Tau (Takami et al. 2002), showing broad emission and a blueshifted absorption feature, confirming the prediction of BEK that the inner wind is traced by helium.

Using Keck II's NIRSPEC, we are undertaking a census of He I $\lambda 10830$ in accreting young stars to evaluate the constraints that helium places on inner winds. In this Letter, we present He I $\lambda 10830$ profiles for six low-mass YSOs with higher-thanaverage disk accretion and mass outflow rates to demonstrate that this feature offers us a new insight into mass loss from young stars with accretion disks.

\section{OBSERVATIONS}

The six YSOs that we have selected to demonstrate the diagnostic potential of He I $\lambda 10830$ are listed in Table 1, along with some outflow and accretion properties and some observed characteristics of their $\lambda 10830$ profiles. These stars are among the CTTSs with the highest disk accretion rates, as determined from their large optical veiling. Most accretion rates from Table 1 are from Hartigan, Edwards, \& Ghandour (1995, hereafter HEG) and are about an order of magnitude higher than the average found for CTTSs in that study, $\dot{M}_{\text {acc }} \sim 10^{-7} M_{\odot} \mathrm{yr}^{-1}$. A more recent analysis of CTTS disk accretion rates (Gullbring et al. 1998) puts the average rate at $\dot{M}_{\text {acc }} \sim 10^{-8} M_{\odot} \mathrm{yr}^{-1}$, but these high accretion rate stars were not included in that analysis. As also noted in Table 1, our sample stars all have collimated jets traced by high-velocity forbidden line emission, and all have inner winds traced by blueshifted absorption at $\mathrm{H} \alpha$. Five (AS 353A, DG Tau, DR Tau, HL Tau, and SVS 13) are extreme Class II sources (Lada 1987), although two of these (HL Tau and SVS 13) are sometimes included in compilations of Class I sources, presumably because of their large extinction and their well-developed jets and molecular outflows. The sixth (DL Tau) is a more typical CTTS. In addition, we have also observed the "weak" T Tauri star (WTTS) V827 Tau, a nonaccreting star of comparable mass and age to the six CTTSs.

Spectra were acquired with NIRSPEC on Keck II in its echelle mode with the N1 filter ( $Y$ band), providing wavelength coverage from 0.95 to $1.12 \mu \mathrm{m}$ at $R=25,000$. Spectra of DG Tau and HL Tau were taken on 2001 November 22 by the Gemini-Keck queue observers T. Geballe and M. Takamiya. The remaining spectra were taken during a three night run in 2002 November by L. Hillenbrand and S. Edwards. Spectra were processed in IRAF and were spatially rectified with a reduction script written and provided by M. Takamiya. Spectra were corrected for telluric emission by subtracting pairs of spectra taken at two positions along the $12^{\prime \prime}$ slit and were corrected for telluric absorption by dividing each spectrum by an early-type standard taken at a similar air mass. During the 2002 run, spectra were acquired without the image derotator because of equipment malfunction. Although this prevents us from studying spatially extended emission, as reported for DG Tau (Takami et al. 2002), it allows us to extract a spectrum from the central point source, where most of the helium emission and all of the helium absorption arise.

\section{DISCUSSION}

The He I $\lambda 10830$ profiles for six high accretion rate CTTSs are shown in Figures 1 and 2. The $\lambda 10830$ profiles all show unequivocal evidence of mass loss with blueshifted absorption penetrating below the continuum level, with depths ranging from 20\% (DG Tau) to 90\% (DR Tau), and with maximum velocities ranging from -200 (HL Tau) to $-450 \mathrm{~km} \mathrm{~s}^{-1}$ (DG Tau). In Figure 1, we compare them with nonsimultaneous profiles for He I $\lambda 5876$ from BEK for the four stars in common with that study, and in Figure 2, we compare them with nonsimultaneous $\mathrm{H} \alpha$ profiles from BEK or L. Hillenbrand \& White (in preparation). While blueshifted absorption is not seen in the $\lambda 5876$ transition (discussed below), it is present in traditional indicators of the inner wind, such as the $\mathrm{H} \alpha$ profiles shown here. However, what distinguishes $\mathrm{He}$ I $\lambda 10830$ is both the prominence and the depth of its blueshifted absorption, offering the promise of a more direct probe of the inner wind. At $\mathrm{H} \alpha$, all profiles are overwhelmingly dominated by broad and strong emission, while for $\lambda 10830$, four of the six stars are either predominantly in absorption (DR Tau and SVS 13) or else resemble classic P Cygni profiles, with comparable levels of emission and absorp- 


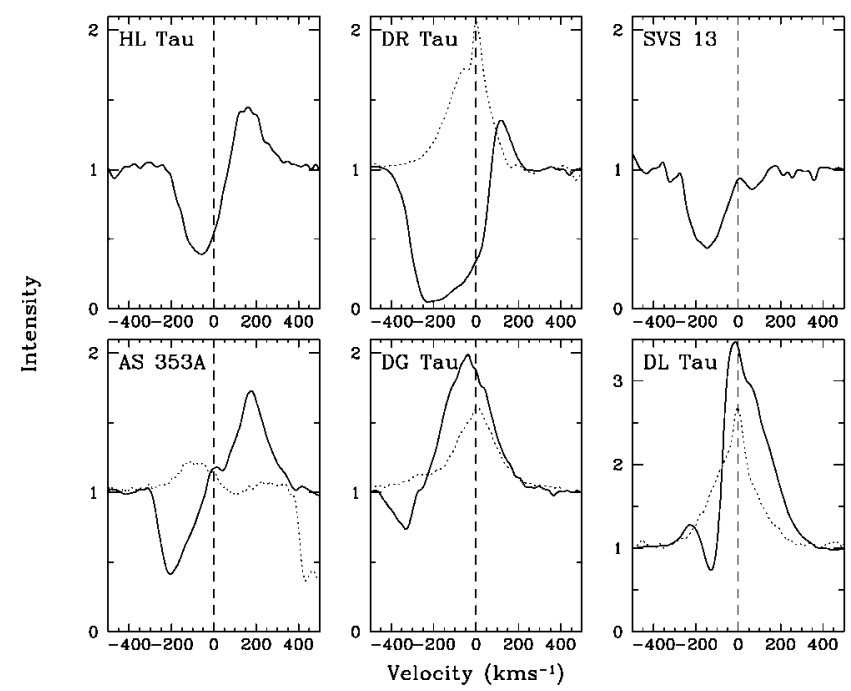

Fig. 1.-Solid lines: He I $\lambda 10830$ profiles for six low-mass young stars with high disk accretion rates. Spectra are normalized to the continuum intensity and registered at the velocity of the ambient molecular cloud, known to be within a few kilometers per second of the stellar velocity for Taurus YSOs (Hartmann et al. 1986). For four of the stars, nonsimultaneous He I $\lambda 5876$ profiles, normalized to their continuum intensity, are also shown as dotted lines.

tion (HL Tau and AS 353A). Moreover, at $10830 \AA$, the blue absorption is far deeper and broader than at $\mathrm{H} \alpha$, penetrating the continuum in all six stars. The most significant aspect of the blueshifted absorption at helium is that in four stars (DR Tau, HL Tau, SVS 13, and AS 353A), it penetrates to depths of more than $50 \%$ into the continuum over a continuous and broad velocity range extending from near the stellar rest velocity to the terminal wind velocity, as inferred from velocities characterizing their spatially extended jets (see Table 1).

The uniqueness of $\mathrm{He}$ I $\lambda 10830$ as a probe of the inner wind derives from the metastability of its lower level $\left(2 s^{3} S^{o}\right)$, which, although energetically far above $(20 \mathrm{eV})$ the singlet ground state, is radiatively isolated from it. Whether this metastable level is populated by recombination and cascade or by collisional excitation from the ground state, it will become significantly populated relative to other excited levels owing to its weak de-excitation rate via collisions to singlet states, making it an ideal candidate to form an absorption line. This absorption is essentially a resonant scattering process since the $\lambda 10830$ transition is the only permitted radiative transition from its upper state to a lower state and since the electron density is unlikely to be so high as to cause collisional excitation or de-excitation. The absence of any absorption or emission at $10830 \AA \AA$ in the nonaccreting WTTS V827 Tau (not shown), which like all WTTSs has strong coronal X-rays (Feigelson et al. 2002), clarifies that the presence of an inner T Tauri wind traced via resonant scattering from the helium metastable level requires the additional presence of disk accretion and its associated energetic phenomena.

New insight regarding the inner wind in CTTSs can be made from visual inspection of the helium profiles for those four stars in which resonance scattering is the dominant formation mechanism. Their deep and broad absorption troughs reveal new information on the kinematics of the inner wind, probing its acceleration region in an accreting star for the first time, and on the geometry of the inner wind, suggesting that the acceleration region is likely close to the star and occults a significant portion of it.

The insight into the kinematics of the wind is provided by

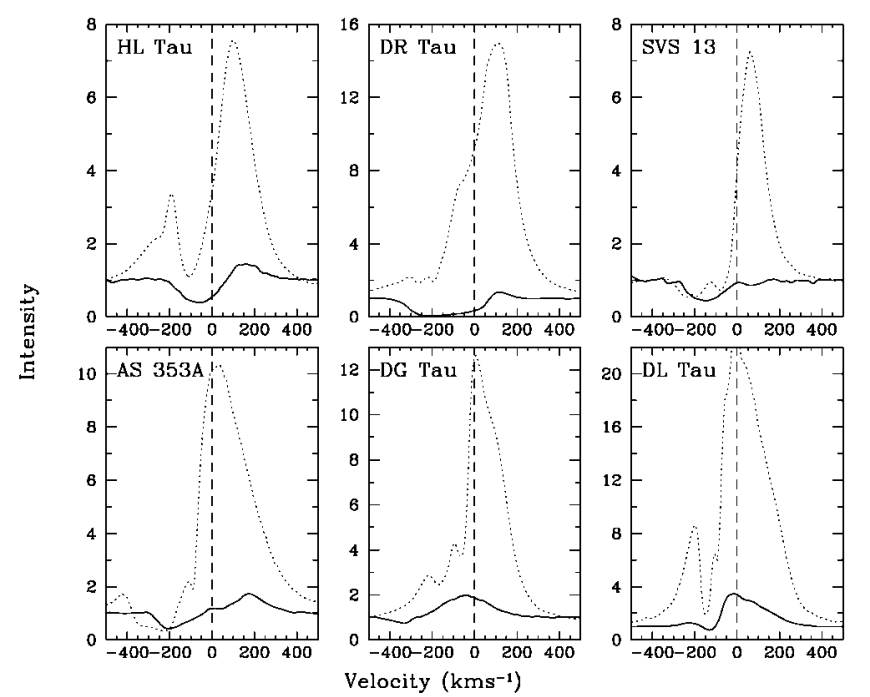

FIG. 2.-Same six He I $\lambda 10830$ profiles from Fig. 1 (solid lines) rescaled for comparison with nonsimultaneous profiles of $\mathrm{H} \alpha$ (dotted lines). At $\mathrm{H} \alpha$, the profiles are overwhelmingly in emission, while at $\mathrm{He} \mathrm{I}$, blueshifted absorption is a far more prominent contributor.

the large and continuous span of velocities over which the blueshifted absorption is observed. This suggests that we are probing the acceleration region of the inner wind, as the absorption extends from velocities at rest relative to the star continuously through to velocities comparable to or often larger than the terminal wind velocities inferred from spatially extended jets. There is also a suggestion of turbulence in the inner wind since in two of the stars, the blueshifted absorption trough extends to velocities as red as $50 \mathrm{~km} \mathrm{~s}^{-1}$.

The insight into the geometry of the inner wind comes from the depth of the absorption into the $1 \mu \mathrm{m}$ continuum over such a wide range of velocities, from rest velocity to the terminal velocity of the wind. In fact, the actual depletion of blueshifted continuum photons will exceed what is observed, as some photons scattered into the line of sight will fall within the velocity range of the blue absorption, making it appear shallower and less extended. At $1 \mu \mathrm{m}$, the continuum in a heavily veiled star will have a larger fraction contributed by the stellar photosphere than at other wavelengths, because the spectral energy distributions of the excess emission arising from both the accretion shock on the stellar surface (Gullbring et al. 1998) and the inner disk (Muzerolle et al. 2003) are rapidly decreasing toward this wavelength while the stellar spectral energy distribution is peaking. For example, at $1 \mu \mathrm{m}$, veiling from an accretion shock at $T \sim 8000 \mathrm{~K}$ will be reduced by a factor of 5 compared with the $V$ band, and veiling from the inner disk with $T \sim 1400 \mathrm{~K}$ will be reduced by a factor of 4 compared with the $K$ band.

We have determined the veiling at $1 \mu \mathrm{m}$ for our high accretion rate stars by comparing the depth of photospheric features in the echelle order centered at $1.075 \mu \mathrm{m}$ with the WTTS V827 Tau. Our veiling estimates, listed in Table 1, indicate that the photosphere contributes between $30 \%$ and $50 \%$ of the $1 \mu \mathrm{m}$ continuum in stars known for much higher veilings in the optical and at $2 \mu \mathrm{m}$ (HEG; Muzerolle et al. 2003). (Photospheric lines could not be detected in SVS 13 because of obscuration by emission lines; however, we estimate that the veiling is $\sim 2$ based on the equivalent width of these lines compared with emission features in the other stars.) With the clarification that a significant fraction of the continuum arises from the star, including both the stellar photosphere and the accretion shock on the stellar surface, 
then the implication of the observed depth and velocity range of the absorption in the four stars where $\lambda 10830$ is predominantly formed by resonance scattering is that the full span of the wind acceleration region lies along the line of sight to the stellar disk.

An unusual feature of the $\lambda 10830$ profiles shown here is that two of the four stars with resonance scattering profiles have significant net negative equivalent widths (see Table 1). This is unexpected in the standard formation scenario for a P Cygni line by resonant scattering, which yields a net zero equivalent width in the absence of stellar occultation, indicating that in these two stars far fewer photons are being scattered into the line of sight than out of it. In the most extreme case, DR Tau, the net equivalent width has a value of $-10.2 \AA$ or, equivalently in velocity units, of $283 \mathrm{~km} \mathrm{~s}^{-1}$. We can demonstrate analytically that this unusual behavior can be accounted for in a resonant scattering scenario if occultation of scattered photons is provided by both the star and the accretion disk. For example, if the disk were to extend into the stellar surface, thereby screening photons scattered from receding material, then in a pole-on system with a spherical isotropically scattering shell at $R=2 R_{*}$, the photons scattered into the line of sight amount to only $40 \%$ of the continuum photons scattered out of the line of sight. This fraction increases to $76 \%$ as the inclination changes from $0^{\circ}$ to $90^{\circ}$. The corresponding net negative equivalent widths would thus be characterized by $60 \%-24 \%$ removal of the continuum, values consistent with the large negative values found here for DR Tau and SVS 13. Moving the scattering surface to different radii does not significantly alter this result, although if the disks of these two stars are truncated at a radius outside the scattering surface, then accounting for their large negative equivalent widths will be a challenge.

Additional perspective on the inner wind comes from comparing optical He I profiles with those of $\lambda 10830$. In Figure 1, we have compared (nonsimultaneous) $\lambda 10830$ and $\lambda 5876$ profiles for the four stars also in the BEK study. For these four high accretion rate stars, BEK concluded that their $\lambda 5876$ emission primarily originates in a wind rather than in funnel flows or accretion shocks. This conclusion was based on the large blueshifted centroids and/or the large blue wing velocities found in the broad component of the $\lambda 5876$ emission coupled with no conspicuous narrow component coming from the accretion shock. This interpretation is strengthened by the unequivocal wind signatures found at $10830 \AA$ in the CTTSs studied here, where the blue wings are now in absorption rather than emission. The absence of blue absorption at $5876 \AA$ is readily understood as being due to the optical thinness of this line in the wind, given the strong radiative decay rate of the lower level of the $\lambda 5876$ transition.

The strength of the emission at $5876 \AA$ also suggests that the helium wind can have a significant optical depth at $10830 \AA$. As this optical depth increases, helium emission will be enhanced over the resonant scattering process, either via recombination/cascade and/or collisional excitation. This effect could then account for the two stars investigated here, DG Tau and DL Tau, which have large positive equivalent widths at $10830 \AA$. They could still have an underlying troughlike absorption from resonance scattering, extending from the stellar rest velocity to the wind terminal velocity as seen in the other for stars, which would be filled in by the additional emission processes in the wind. Since both resonant scattering and line emission contribute to $\lambda 10830$ formation, while the primary contributor to $\lambda 5876$ is line emission, we anticipate that simultaneous observations of both He I lines, together with theoretical modeling, may yield the velocity, density, and temperature structures of the inner wind.

In summary, we conclude that the He I $\lambda 10830$ line offers a unique new probe of inner winds in accretion disk systems, revealing the wind acceleration region and providing constraints on the geometry of this region. Most importantly, the deep and broad absorption seen in the four stars seems to require that the full span of the wind acceleration region is seen in projection against the stellar disk. We find it more straightforward to interpret this finding in a scenario in which the wind arises from stellar coronal regions, since it would be difficult for wind material arising from a disk to absorb the radiation from the stellar disk at all velocities, from rest to the terminal wind speed. If this is the case, it would not be a normal "stellar wind" since this phenomenon is strongest in stars with the highest disk accretion rates. We anticipate a fuller understanding of the physical properties of the inner wind, its geometry, and its launch distance from the star when the behavior of this line has been examined for a large sample of accreting young stars covering a wide range of disk accretion rates.

Heartfelt thanks to an anonymous referee who spurred significant improvements in the manuscript and a deep mahalo to the queue observers for the Keck-Gemini NIRSPEC program, Tom Geballe and Marianne Takamiya for the 2001 data. NASA grant NAG5-12996 issued through the Office of Space Science provides support for this project.

\section{REFERENCES}

Ardila, D., Basri, G., Walter, F., Valenti, J., \& Johns-Krull, C. 2002, ApJ, 566, 1100

Beristain, G., Edwards, S., \& Kwan, J. 2001, ApJ, 551, 1037 (BEK)

Calvet, N. 1997, in IAU Symp. 182, Herbig-Haro Flows and the Birth of Low Mass Stars, ed. B. Reipurth \& C. Bertout (Dordrecht: Kluwer), 417

Calvet, N., Hartmann, L., Kenyon, S. J., \& Whitney, B. A. 1994, ApJ, 434, 330

Davis, C. J., Ray, T. P., Desroches, L., \& Aspin, C. 2001, MNRAS, 326, 524

Davis, C. J., Whelan, E., Ray, T. P., \& Chrysostomou, A. 2003, A\&A, 397, 693

Dupree, A. K., Sasselov, D. D., \& Lester, J. B. 1992, ApJ, 387, L85

Edwards, S., \& Snell, R. 1982, ApJ, 261, 151

Feigelson, E. D., Broos, P., Gaffney, J. A., III, Garmire, G., Hillenbrand, L. A., Pravado, S. H., Townsley, L., \& Tsuboi, Y. 2002, ApJ, 574, 258

Gullbring, E., Hartmann, L., Briceño, C., \& Calvet, N. 1998, ApJ, 492, 323 Hartigan, P., Edwards, S., \& Ghandour, L. 1995, ApJ, 452, 736 (HEG)

Hartmann, L., Hewett, R., \& Calvet, N. 1994, ApJ, 426, 669

Hartmann, L., Hewett, R., Stahler, S., \& Mathieu, R. D. 1986, ApJ, 309, 275

Hirose, S., Uchida, Y., Shibata, K., \& Matsumoto, R. 1997, PASJ, 49, 193
Königl, A., \& Pudritz, R. 2000 in Protostars and Planets IV, ed. V. Mannings, A. P. Boss, \& S. S. Russell (Tucson: Univ. Arizona Press), 759

Kwan, J., \& Tademaru, E. 1988, ApJ, 332, L41

Lada, C. J. 1987, in Star Forming Regions, ed M. Peimbert \& J. Jugaku (Dordrecht: Reidel), 1

Mundt, R., Buehrke, T., Solf, J., Ray, T. P., \& Raga, A. C. 1990, A\&A, 232, 37

Muzerolle, J., Calvet, N., \& Hartmann, L. 2001, ApJ, 550, 944

Muzerolle, J., Calvet, N., \& Hartmann, L., \& D'Alessio, P. 2003, ApJ, 597, L149

Najita, J. R., Edwards, S., Basri, G., \& Carr, J. 2000, in Protostars and Planets IV, ed. V. Mannings, A. P. Boss, \& S. S. Russell (Tucson: Univ. Arizona Press), 457

Shu, F., Najita, J., Ostriker, E., Wilkin, F. Ruden, S., \& Lizano, S. 1994, ApJ, 429, 781

Snell, R., \& Edwards, S. 1981, ApJ, 251, 103

Takami, M., Chrysostomou, A., Bailey, J., Gledhill, T., Tamura, M., \& Terada, H. 2002, ApJ, 568, L53

Ulrich, R. K., \& Wood, B. C. 1981, ApJ, 244, 147 